\title{
Soluble Polyimides Based on 2,3,5-Tricarboxycyclopentyl Acetic Dianhydride
}

\author{
Yusuke Tsuda, Yukio Tanaka, Kichinosuke Kamata, Norihiko Hiyoshi,* \\ Shuntaro MataKa, ${ }^{* *}$ Yasuo Matsuki, ${ }^{* * *}$ Michinori NishiKaWA, ${ }^{* * *}$ \\ Shigeo Kawamura, ${ }^{* * *}$ and Nobuo Bessho*** \\ Department of Industrial Chemistry, Kurume National College of Technology, \\ 1232 Komorino-machi, Kurume-shi, Fukuoka 830, Japan \\ * Advanced Engineering School, Kurume National College of Technology, \\ 1232 Komorino-machi, Kurume-shi, Fukuoka 830, Japan \\ ** Institute of Advanced Material, Kyushu University, 6-1 Kasuga-Koh-en, \\ Kasuga-shi, Fukuoka 816, Japan \\ *** Yokkaichi Research Laboratories, Japan Synthetic Rubber Co., Ltd., \\ 100 Kawajiri-cho, Yokkaichi-shi, Mie 510, Japan
}

(Received November 19, 1996)

\begin{abstract}
Soluble polyimides with polyalicyclic structure were prepared by reactions of 2,3,5-tricarboxycyclopentyl acetic dianhydride (TCA-AH) with aromatic diamines. The two-step polymerization systems including poly(amic acids) synthesis and solution imidization using pyridine and acetic anhydride were performed and soluble polyimides with high inherent viscosities and high imidization ratios were obtained. TCA-AH polyimides exhibited high thermal stability in air and nitrogen, and good solubility in common polar solvents such as NMP ( $N$-methyl-2-pyrrolidone). In comparison with soluble aromatic polyimides based on fluorine containing monomers, TCA-AH polyimides showed slightly less thermal stability and lower solubility in a few solvents.

KEY WORDS Polyimide / Soluble Polyimide / Polyalicyclic Structure / 2,3,5-Tricarboxycyclopentyl Acetic Dianhydride / Solution Imidization / Thermal Stability / Solubility /
\end{abstract}

Polyimides exhibit excellent thermal and mechanical properties, and have extensive engineering and microelectronics applications. ${ }^{1}$ Aromatic polyimides such as pyromellitic polyimides are prepared from aromatic diamines and aromatic tetracarboxylic acids via poly(amic acids). Since conventional aromatic polyimides are insoluble, these polymers are usually processed in corresponding soluble poly(amic acid) precursors, and then thermally or chemically imidized. However, there are some problems owing to the unstability of poly(amic acid) and the liberation of water in imidization process. Therefore, solvent soluble polyimides which are processed without difficulty have been desired. Several approaches to improve the solubility of the polyimides have been investigated and successful examples involve the incorporation of fluorine moieties ${ }^{2-6}$ or, chlorine moieties ${ }^{7}$ or, bulky side groups ${ }^{8}$ or, pendant phenyl group $^{9}$ or, polydimethylsiloxane segment ${ }^{10}$ or, polyalicyclic structure ${ }^{1-13}$ into the polymer backbone.

This paper reports the synthesis and characterization of polyimides prepared from a polyalicyclic tetracarboxylic dianhydride, 2,3,5-tricarboxy-cyclopentyl acetic dianhydride (TCA-AH) and aromatic diamines. Although soluble polyimides using TCA-AH have been widely used as alignment films for liquid crystal displays, ${ }^{14-16}$ fundamental studies on these polyimides have not reported in detail. Therefore, we focused on the effects of TCA-AH on solubility of polyimides and parameters such as inherent viscosities, imidization ratio, thermal stability, and solubility in various solvents. Conventional aromatic dianhydrides such as pyromellitic dianhydride (PMDA), 3,3',4,4'-benzophenonetetracarboxylic dianhydride (BTDA) and 3,3',4,4'-biphenyltetracarboxylic dianhydride (BPDA), and 4,4'-Hexafluoroisopropylidenedi (phthalic anhydride) (6FDA) known to give a soluble polyimide by the effect of two trifluoro methyl groups were used as references.

\section{EXPERIMENTAL}

\section{Materials}

TCA-AH (mp $194-195^{\circ} \mathrm{C}$ from acetic anhydride) and 3,5-diamino- $3^{\prime}$-trifluoromethyl benzanilide (DTBA) (mp $195-196^{\circ} \mathrm{C}$ from ethanol/water mixture) were kindly supplied by Japan Synthetic Rubber Co., Ltd. PMDA (Mitsubishi Gas Chemical), BTDA (Mitsui Toatsu Chemicals Inc.), BPDA (Ube Industries, Ltd.), 6FDA (Central Glass Co., Ltd.), 1,4-phenylenediamine (PDA) (Wakayama Seika Kogyo Co., Ltd.), 4,4'-diaminodiphenylmethane (DDM) (Mitsui Toatsu Chemicals Inc.), 4,4'-diaminodiphenylether (DDE) (Wakayama Seika Kogyo Co., Ltd.), and 2,2-bis(4-aminophenyl)hexafluoropropane (BIS-A-AF) (Central Glass Co., Ltd.) were used as received. $N$-Methyl-2-pyrrolidone (NMP) (Mitsubishi Kasei) were distilled under reduced pressure. Reagent grade $N, N$-dimethylformamide (DMF), $N, N$-dimethylacetamide (DMAC), dimethyl sulfoxide (DMSO), 1,3-dimethyl-2-imidazolidinone (DMI), $m$ cresol, tetrahydrofuran (THF), dichloromethane, sulfuric acid, acetic anhydride, and pyridine were purchased from Katayama Chemical Industries Co., Ltd. and used as received. Dimethyl sulfoxide- $d_{6}$ (Aldrich Chem. Co.) was used as received.

\section{Measurement}

The inherent viscosities of all polymers were measured using Cannon Fenske viscometers at a concentration of $0.5 \mathrm{~g} \mathrm{dL}^{-1}$ in NMP at $30^{\circ} \mathrm{C}$. Thermogravimetric analysis (TGA) was performed on a Shimadzu thermogravimetric analyzer Model TGA-50 in air or nitrogen at a heating 
rate of $10^{\circ} \mathrm{C} \mathrm{min}{ }^{-1} \cdot{ }^{1} \mathrm{H}$ NMR spectra were measured on a JEOL JNM-EX270 FT NMR in a dimethyl sulfoxide$d_{6}$ with tetramethylsilane (TMS) as an internal reference. IR spectra were measured on a JASCO IR Report-100 spectrophotometer.

\section{Poly(amic acid) Preparations}

As a typical example, to a $30-\mathrm{mL}$ flask were added $0.792 \mathrm{~g}(3.53 \mathrm{mmol})$ of TCA-AH and $0.708 \mathrm{~g}(3.53 \mathrm{mmol})$ of DDE. The system was purged by nitrogen and NMP was added. Monomer concentration was kept at 10 $15 \mathrm{wt} \%$. The mixture was stirred at $60^{\circ} \mathrm{C}$ under nitrogen for 12 hours to allow viscosity to increase. Additional NMP was added to the mixture to keep the polymer concentration $5-10 \mathrm{wt} \%$ and about one-third portions of the mixture were poured into a large amount of water or methanol. Precipitated poly(amic acid) was filtered, washed with excess methanol and dried at room temperature for 2 days. The residual two-third portions of the poly(amic acid) solution were used for the following polyimide preparation. The IR spectrum $(\mathrm{KBr})$ exhibited absorptions at $3300 \mathrm{~cm}^{-1}(\mathrm{O}-\mathrm{H}$ and $\mathrm{N}-\mathrm{H}), 1715$ and $1660 \mathrm{~cm}^{-1}(\mathrm{C}=\mathrm{O})$. Chemical shifts of the characteristic absorption peaks of ${ }^{1} \mathrm{H}$ NMR spectra and results of the elemental analyses are as follows. ${ }^{1} \mathrm{H}$ NMR (DMSO): $\delta$ $(\mathrm{ppm})=c a .1 .8-4.0(\mathrm{~m}, 8 \mathrm{H}$, aliph. $), 6.85-7.03(\mathrm{~m}, 4 \mathrm{H}$, arom.), 7.47-7.67 (m, 4H, arom.), 9.87-10.16 (m, 2H, $-\mathrm{NH}-)$. Anal. Calcd for $\left(\mathrm{C}_{22} \mathrm{H}_{20} \mathrm{~N}_{2} \mathrm{O}_{7}\right)_{n}: \mathrm{C}, 62.26 \% ; \mathrm{H}$, $4.75 \%$; N, 6.60\%. Found: C, 60.05\%; H, 5.12\%; N, $6.57 \%$. The carbon analysis value was found to be lower than the calculated value and a trace amount of residue was observed after elemental analysis, probably due to the formation of char characteristic of heat resistant polymers. $^{17}$

\section{Polyimide Preparations}

As a typical example, to $10 \mathrm{~g}$ of $10 \mathrm{wt} \% \mathrm{TCA}-\mathrm{AH} /$ DDE poly(amic acid) solution were added $1 \mathrm{~g}$ of pyridine and $1 \mathrm{~g}$ of acetic anhydride. The system was purged by nitrogen and was stirred at $110-120^{\circ} \mathrm{C}$ under nitrogen for $4-7$ hours. Powdered polyimides were obtained by precipitation into a large amount of methanol, filtered, and washed with a large amounts of methanol, and dried at $100^{\circ} \mathrm{C}$ for 1 day. The IR spectrum (KBr) exhibited absorptions at 1735 and $1690 \mathrm{~cm}^{-1}(\mathrm{C}=\mathrm{O})$, $1360 \mathrm{~cm}^{-1}(\mathrm{C}-\mathrm{N})$. Chemical shifts of the characteristic absorption peaks of ${ }^{1} \mathrm{H}$ NMR spectra and the results of the elemental analyses are as follows. ${ }^{1} \mathrm{H}$ NMR (DMSO): $\delta(\mathrm{ppm})=c a .1 .8-3.9(\mathrm{~m}, 8 \mathrm{H}$, aliph.), 6.857.31 (m, 6H, arom.), 7.48-7.71 (m, 2H, arom.), 9.9110.24 (m, unreacted - NH-). Detailed descriptions of ${ }^{1} \mathrm{H}$ NMR spectra are given in next section. Anal. Calcd for $\left(\mathrm{C}_{22} \mathrm{H}_{16} \mathrm{~N}_{2} \mathrm{O}_{5}\right)_{n}$ : C, 68.04\%; $\mathrm{H}, 4.15 \%$; N, $7.21 \%$. Found: C, $64.74 \% ; \mathrm{H}, 4.81 \%$; N, $7.13 \%$. The carbon analysis value was found to be lower than the calculated value and a trace amount of residue was observed after the elemental analysis.

\section{RESULTS AND DISCUSSION}

The synthetic route of the polyimides from TCA$\mathrm{AH}$ and the various aromatic diamines is illustrated in Scheme 1. Two-step polymerization systems including
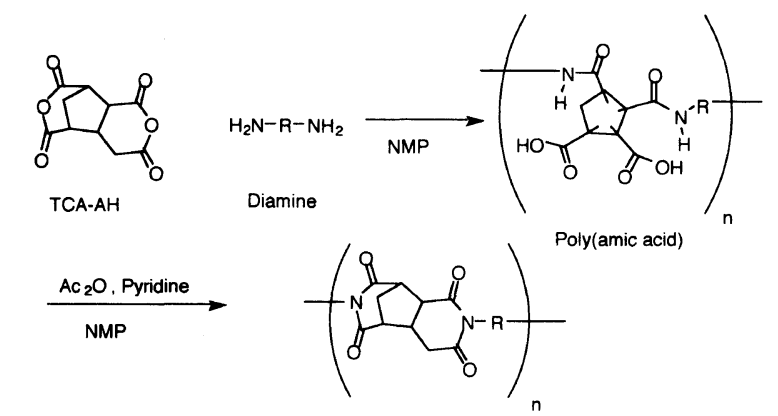

Polyimide
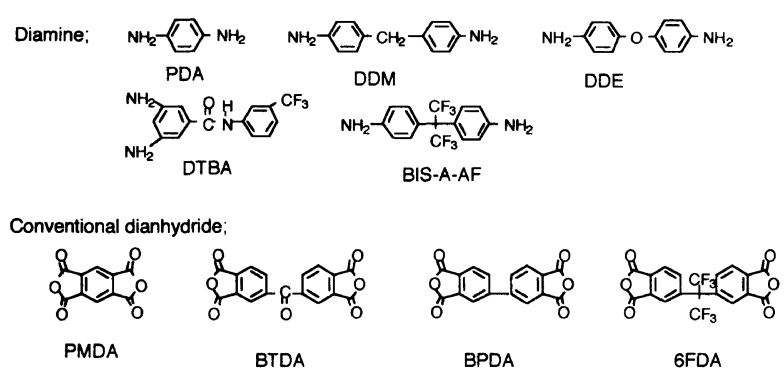

Scheme 1. Synthesis of polyimides based on 2,3,5-tricarboxycyclopenthyl acetic dianhydride (TCA-AH).

poly(amic acids) synthesis and solution imidization process were performed. The poly(amic acids) based on TCA-AH theoretically contain four isomers due to unsymmetrical structure of TCA-AH. The poly(amic acids) were obtained by reacting TCA-AH with an equimolar amount of PDA, DDM, DDE, DTBA, or BIS-A-AF at $60^{\circ} \mathrm{C}$ for 12 hours under a nitrogen atmosphere. The polyimides were obtained by solution imidization at $110-120^{\circ} \mathrm{C}$ in the presence of pyridine as a base catalyst and acetic anhydride as a dehydrating agent. Conventional aromatic tetracarboxylic dianhydrides such as PMDA, BTDA, and BPDA, as well as 6FDA containing two trifluoro methyl groups were also reacted with PDA, DDM, DDE, DTBA, and BIS-A-AF under the same manner as TCA-AH. The experimental results of all combinations of above dianhydrides and diamines are summarized in Table I. Out of 25 combinations studied, 12 NMP soluble polyimides were obtained. In these cases, clear polyimide solutions were eventually obtained. In other cases, clear polyamic acid solutions were obtained. However, gelation or precipitation happened in the course of imidization process. TCA-AH gave NMP soluble polyimides regardless of diamine structure and thus the effects of TCA-AH on the enhancement of solubility were confirmed. The inherent viscosities of TCA-AH polyimides based on conventional aromatic diamines such as PDA, DDM, and DDE were high in the range of $0.62-0.66 \mathrm{dL} \mathrm{g}^{-1}$. Those based on DTBA and BIS-A-AF were low in the range of $0.16-0.32$ $\mathrm{dL} \mathrm{g}^{-1}$. These differences are probably due to the low reactivity of these diamines, which have electron withdrawing groups such as trifluoromethyl group and amido carbonyl group.

The polymerization conditions of poly(amic acids) were investigated in detail. Table II shows the dependence of poly(amic acids) polymerization conditions on the inherent viscosities which are the estimate of molecular weight. This poly(amic acids) synthesis is the key 
Table I. Poly(amic acids) and corresponding polyimides using various combinations of tetracarboxylic dianhydrides and aromatic diamines

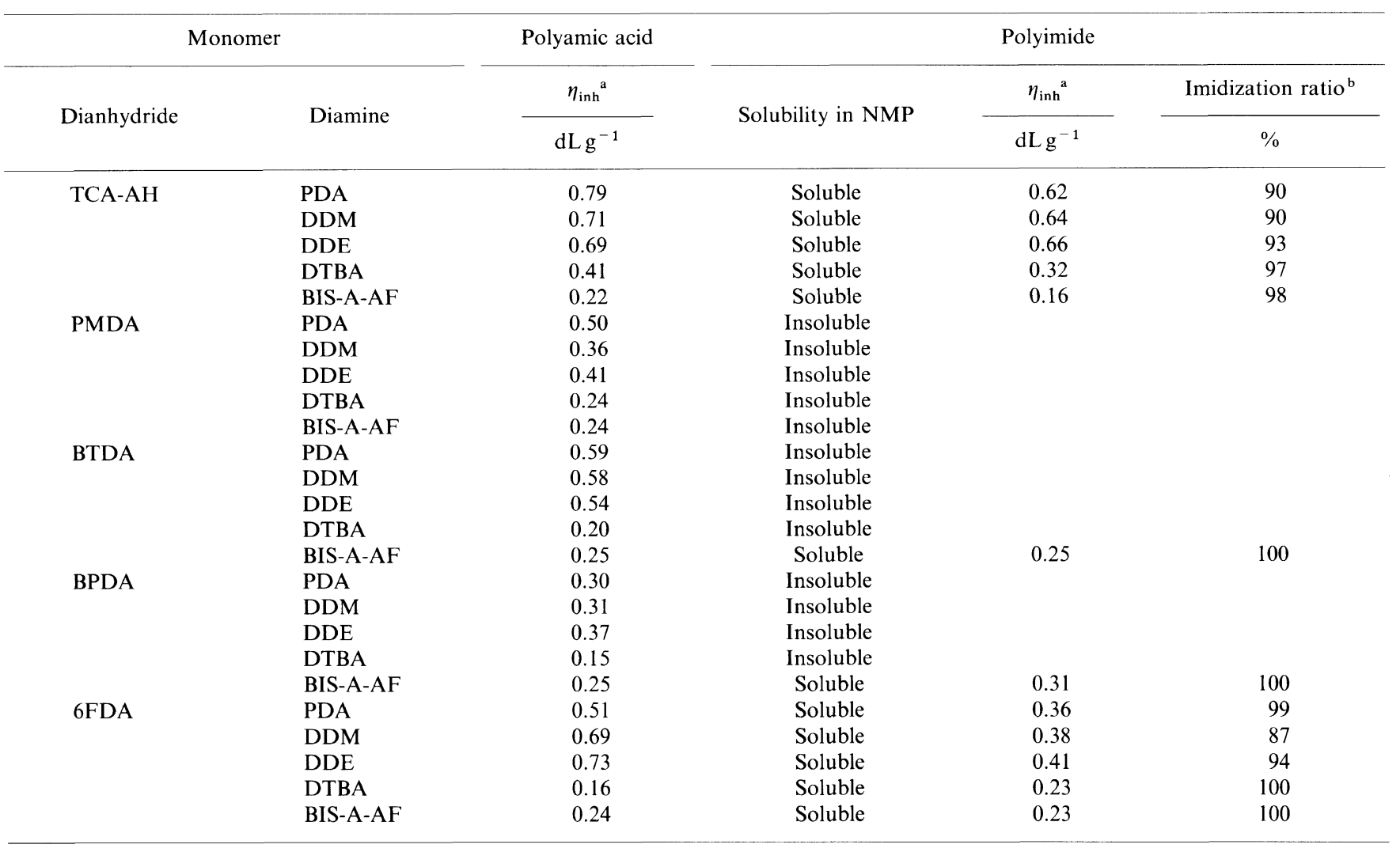

${ }^{a}$ Measured at $0.5 \mathrm{~g} \mathrm{dL}^{-1}$ in NMP at $30^{\circ} \mathrm{C} .{ }^{\mathrm{b}}$ Calculated from ${ }^{1} \mathrm{H}$ NMR measurement.

Table II. Poly(amic acids) synthesis using TCA-AH with aromatic diamines under various conditions

\begin{tabular}{|c|c|c|c|c|}
\hline \multirow{3}{*}{$\frac{\text { Monomer }^{\mathrm{a}}}{\text { Diamine }}$} & \multicolumn{3}{|c|}{ Polymerization conditions } & \multirow{3}{*}{$\begin{array}{c}\text { Polyamic acids } \\
\frac{\eta_{\mathrm{inh}}{ }^{\mathrm{b}}}{\mathrm{dL} \mathrm{g}^{-1}}\end{array}$} \\
\hline & \multirow{2}{*}{$\begin{array}{c}\text { Concentration } \\
\mathbf{0}\end{array}$} & \multirow{2}{*}{$\frac{\text { Temperature }}{{ }^{\circ} \mathrm{C}}$} & \multirow{2}{*}{$\frac{\text { Time }}{\mathrm{h}}$} & \\
\hline & & & & \\
\hline PDA & 15 & 60 & 12 & 1.09 \\
\hline PDA & 15 & 30 & 12 & 0.49 \\
\hline PDA & 10 & 60 & 12 & 0.80 \\
\hline DDM & 15 & 60 & 12 & 1.00 \\
\hline DDM & 15 & 30 & 12 & 0.73 \\
\hline DDM & 10 & 30 & 12 & 0.42 \\
\hline DDE & 15 & 60 & 2 & 0.48 \\
\hline $\mathrm{DDE}$ & 15 & 60 & 4 & 0.61 \\
\hline DDE & 15 & 60 & 12 & 1.01 \\
\hline $\mathrm{DDE}$ & 10 & 60 & 12 & 0.79 \\
\hline
\end{tabular}

${ }^{a}$ Equimolar amount of TCA-AH was used. ${ }^{b}$ Measured at $0.5 \mathrm{~g} \mathrm{dL}^{-1}$ in NMP at $30^{\circ} \mathrm{C}$.

step which controls the molecular weight of the resulting polyimides. No significant changes of molecular weight occur during imidization, although the virtual inherent viscosities of polyimides are reduced from the value of poly(amic acid)s (Table I). Three significant factors which control inherent viscosity are monomer concentration in NMP, polymerization temperature and polymerization time. The higher monomer concentrations gave poly(amic acids) with higher inherent viscosities. However, the solutions with higher solid content were very viscous and difficult to handle. Therefore, $10-15 \mathrm{wt} \%$ was the most optimum concentration. $60^{\circ} \mathrm{C}$ was suitable temperature to obtain higher molecular weight polymers. Above $60^{\circ} \mathrm{C}$ there was unfavorable coloration. Longer reaction time gave polymers with higher inherent vis- cosities. Over 12 hours no significant change occurred.

High imidization ratios are required to obtain stable polyimides with excellent performance. Nishikawa suggests that the imidization ratios of soluble polyimides based on TCA-AH strongly affect the alignment of liquid crystals when used as a alignment film for liquid crystal display. ${ }^{14}$ The imidization ratios of TCA-AH polyimides were measured by ${ }^{1} \mathrm{H}$ NMR and calculated from the reduction of intensity ratio of the $\mathrm{NH}$ proton signals in poly(amic acids) to the aromatic proton signals. Figure 1 shows a representative ${ }^{1} \mathrm{H}$ NMR spectra of TCAAH/DDE polyimides. Broad signals of the $\mathrm{NH}$ proton appears 10.0 to $10.2 \mathrm{ppm}$, while a series of complicated aromatic proton resonances appears 6.9 to $7.7 \mathrm{ppm}$. Thus, imidization ratios were calculated by the fol- 




TCA-AH / DDE Polyimide

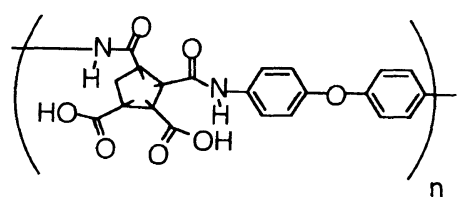

TCA-AH / DDE Poly(amic acid)

$$
\begin{aligned}
& \frac{\text { Intensity ratio of } \mathrm{NH} \text { protons }}{\text { Intensity ratio of all aromatic protons }}=\frac{1-\mathrm{X} / 100}{4} \\
& \mathrm{X} \text {; imidization ratio }(\%) .
\end{aligned}
$$



Figure 1. ${ }^{1} \mathrm{H}$ NMR spectra of representative TCA-AH polyimide (TCA-AH/DDE).

lowing equation; intensity ratio of $\mathrm{NH}$ protons/intensity ratio of all aromatic protons $=(1-X / 100) / 4$, where $X$ is imidization ratio $(\%)$. The imidization ratios of TCA$\mathrm{AH}$ polyimides were sufficiently high and in the range of $90-97 \%$.

6FDA also gave NMP soluble polyimides with high imidization ratios in all combinations (Table I). Although the inherent viscosities of 6FDA polyimides were lower than those of corresponding TCA-AH polyimides in most cases, these conditions might not be optimized for 6FDA polyimides. Therefore, the effect of TCA-AH on the molecular weight of polyimides may not be compared with that of 6FDA in these experiments.

From the standpoint of the diamine structures, conventional aromatic diamines such as PDA, DDM, and DDE do not give soluble polyimides except with TCAAH or 6FDA. BIS-A-AF containing two trifluoro methyl groups gave NMP soluble polyimides except with the most rigid tetracarboxylic dianhydride, PMDA. DTBA behave the same as other conventional aromatic diamines even though possessing a trifluoro methyl group. This difference is based on the fact that DTBA contains only single trifluoro methyl group, and the rigid amide linkage in DTBA reduce the solubility.

TCA-AH polyimides showed good thermal stability in air and nitrogen as revealed by TGA (Table III). Figure 2 shows typical TGA curves of soluble polyimide using TCA-AH and PDA as monomers. All the polymers indicated no weight loss below $350^{\circ} \mathrm{C}$ in air, and $10 \%$ weight loss temperatures were in the range of $422-561^{\circ} \mathrm{C}$ in air and $457-575^{\circ} \mathrm{C}$ in nitrogen. TCA-AH polyimides show about $100^{\circ} \mathrm{C}$ lower thermal stability compared with aromatic soluble polyimides using fluorine containing

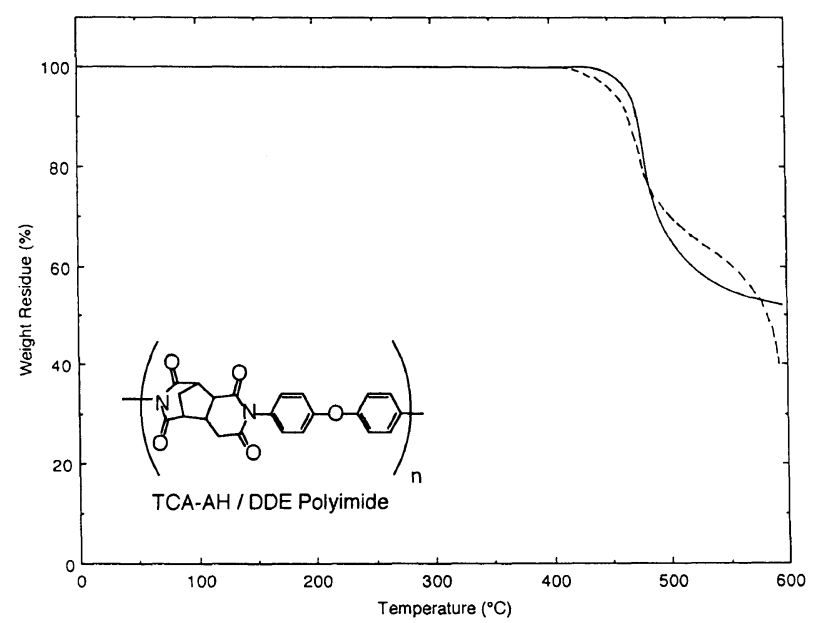

Figure 2. TGA curves of representative TCA-AH polyimide (TCAAH/PDA) in nitrogen (real line) and in air (dotted line).

monomers such as BIS-A-AF and 6FDA (Table III). These differences are probably due to the aliphatic structure of TCA-AH. TCA-AH polyimides still can be ranked as heat resistant polymers.

The solubilities of the obtained polyimides were determined in 9 common solvents at $5 \mathrm{wt} \%$ in NMP (Table IV). TCA-AH polyimides were soluble in polar solvents such as NMP, DMF, DMAC, DMSO, DMI, and $\mathrm{H}_{2} \mathrm{SO}_{4}$. In $m$-cresol, most TCA-AH polyimides were soluble except polyimides based on the most rigid PDA, while in THF, most TCA-AH polyimides were insoluble except polyimides based on BIS-A-AF with two trifluoro methyl groups. 6FDA polyimides showed better solubility in $m$-cresol, THF and dichloromethane 
Table III. Decomposition temperature of soluble polyimides

\begin{tabular}{|c|c|c|c|c|c|}
\hline \multicolumn{4}{|c|}{ Polyimide } & \multicolumn{2}{|c|}{$10 \%$ Weight loss temperature ${ }^{c}$} \\
\hline \multirow{2}{*}{ Dianhydride } & \multirow{2}{*}{ Diamine } & $\eta_{\text {inh }}{ }^{\mathrm{a}}$ & Imidization ratio ${ }^{b}$ & in Air & in Nitrogen \\
\hline & & $\mathrm{dLg}^{-1}$ & $\%$ & ${ }^{\circ} \mathrm{C}$ & ${ }^{\circ} \mathrm{C}$ \\
\hline \multirow[t]{5}{*}{ TCA-AH } & PDA & 0.62 & 90 & 466 & 474 \\
\hline & DDM & 0.64 & 90 & 470 & 474 \\
\hline & DDE & 0.66 & 93 & 422 & 457 \\
\hline & DTBA & 0.32 & 97 & 452 & 494 \\
\hline & BIS-A-AF & 0.16 & 98 & 432 & 478 \\
\hline BTDA & BIS-A-AF & 0.25 & 100 & 557 & 575 \\
\hline BPDA & BIS-A-AF & 0.31 & 100 & 560 & 573 \\
\hline \multirow[t]{4}{*}{$6 \mathrm{FDA}$} & PDA & 0.36 & 99 & 554 & 572 \\
\hline & DDM & 0.38 & 87 & 533 & 558 \\
\hline & DDE & 0.41 & 94 & 561 & 571 \\
\hline & DTBA & 0.23 & 100 & 496 & 524 \\
\hline
\end{tabular}

${ }^{a}$ Measured at $0.5 \mathrm{~g} \mathrm{dL}^{-1}$ in NMP at $30^{\circ} \mathrm{C}$. ${ }^{\mathrm{b}}$ Calculated from ${ }^{1} \mathrm{H}$ NMR measurement. ${ }^{\mathrm{c}}$ Measured at a heating rate of $10^{\circ} \mathrm{C}$ min ${ }^{-1}$.

Table IV. Solubility behavior of polyimides in various solvents

Polyimide

Solubility $^{\mathrm{c}}$

\begin{tabular}{|c|c|c|c|c|c|c|c|c|c|c|c|c|}
\hline \multirow{2}{*}{ Dianhydride } & \multirow{2}{*}{ Diamine } & $\eta_{\text {inh }}{ }^{a}$ & Imidization ratio $^{b}$ & \multirow{2}{*}{ NMP } & \multirow{2}{*}{ DMF } & \multirow{2}{*}{ DMAC } & \multirow{2}{*}{ DMSO } & \multirow{2}{*}{ DMI } & \multirow{2}{*}{$m$-Cresol } & \multirow{2}{*}{ THF } & \multirow{2}{*}{$\mathrm{CH}_{2} \mathrm{Cl}_{2}$} & \multirow{2}{*}{$\mathrm{H}_{2} \mathrm{SO}_{4}$} \\
\hline & & $\mathrm{dLg}^{-1}$ & $\%$ & & & & & & & & & \\
\hline \multirow[t]{5}{*}{ TCA-AH } & PDA & 0.62 & 90 & $\mathrm{~S}$ & $\mathrm{~S}$ & $\mathrm{~S}$ & $\mathrm{~S}$ & $\mathrm{~S}$ & I & I & I & $\mathrm{S}$ \\
\hline & DDM & 0.64 & 90 & $\mathrm{~S}$ & $\mathrm{~S}$ & $\mathrm{~S}$ & $\mathrm{~S}$ & $\mathrm{~S}$ & $\mathrm{~S}$ & I & I & $\mathrm{S}$ \\
\hline & DDE & 0.66 & 93 & $\mathrm{~S}$ & $\mathrm{~S}$ & $\mathrm{~S}$ & $\mathrm{~S}$ & $\mathrm{~S}$ & $\mathrm{~S}$ & I & I & $\mathrm{S}$ \\
\hline & DTBA & 0.32 & 97 & $\mathrm{~S}$ & $S$ & $\mathrm{~S}$ & $\mathrm{~S}$ & $\mathrm{~S}$ & $\mathrm{~S}$ & I & I & $\mathrm{S}$ \\
\hline & BIS-A-AF & 0.16 & 98 & $\mathrm{~S}$ & $\mathrm{~S}$ & $\mathrm{~S}$ & $\mathrm{~S}$ & $\mathrm{~S}$ & $\mathrm{~S}$ & $\mathrm{~S}$ & I & $\mathrm{S}$ \\
\hline BPDA & BIS-A-AF & 0.31 & 100 & $\mathrm{~S}$ & $\mathrm{~S}$ & I & $\mathrm{S}(\mathrm{h})$ & $\mathrm{S}(\mathrm{h})$ & $\mathbf{S}$ & $\mathrm{S}$ & $\mathrm{S}$ & $\mathrm{S}$ \\
\hline \multirow[t]{5}{*}{$6 \mathrm{FDA}$} & PDA & 0.36 & 99 & $\mathrm{~S}$ & $\mathrm{~S}$ & $\mathrm{~S}$ & $\mathrm{~S}$ & $\mathrm{~S}$ & $\mathrm{~S}$ & I & $\mathrm{S}$ & $\mathrm{S}$ \\
\hline & DDM & 0.38 & 87 & $\mathrm{~S}$ & $\mathrm{~S}$ & $S$ & $\mathrm{~S}$ & $\mathrm{~S}$ & $\mathrm{~S}$ & $\mathrm{~S}$ & $\mathrm{~S}$ & $\mathrm{~S}$ \\
\hline & DDE & 0.41 & 94 & $\mathrm{~S}$ & $\mathrm{~S}$ & $\mathrm{~S}$ & $\mathrm{~S}$ & $\mathrm{~S}$ & $\mathrm{~S}$ & $\mathrm{~S}$ & $\mathrm{~S}$ & $\mathrm{~S}$ \\
\hline & DTBA & 0.23 & 100 & $\mathrm{~S}$ & $\mathrm{~S}$ & $\mathrm{~S}$ & $\mathrm{~S}$ & $\mathrm{~S}$ & $\mathrm{~S}$ & $\mathrm{~S}$ & I & $\mathrm{S}$ \\
\hline & BIS-A-AF & 0.23 & 100 & $\mathrm{~S}$ & $\mathrm{~S}$ & $\mathrm{~S}$ & $\mathrm{~S}$ & $\mathrm{~S}$ & $\mathrm{~S}$ & $\mathrm{~S}$ & $\mathrm{~S}$ & $\mathrm{~S}$ \\
\hline
\end{tabular}

${ }^{a}$ Measured at $0.5 \mathrm{~g} \mathrm{dL}^{-1}$ in NMP at $30^{\circ} \mathrm{C} .{ }^{\mathrm{b}}$ Calculated from ${ }^{1} \mathrm{H}$ NMR measurement. ${ }^{\mathrm{c}} \mathrm{S}$, soluble; S(h), soluble at a heated temperature; I, insoluble.

than TCA-AH polyimides. In these solvents, the flexible isopropyl group and two trifluoro methyl groups in 6FDA seem to be more effective than the polyalicyclic structure in TCA-AH.

\section{CONCLUSIONS}

Soluble polyimides with polyalicyclic structures were prepared by the reaction of TCA-AH with aromatic diamines. The two-step polymerization systems including poly(amic acids) synthesis and solution imidization using pyridine and acetic anhydride were performed. To obtain high molecular weight polymers, optimum polymerization conditions were $60^{\circ} \mathrm{C}, 12$ hours polymerization time and $10-15 \mathrm{wt} \%$ monomer concentration in NMP. TCAAH polyimides exhibited good thermal stability and $10 \%$ weight loss temperature was in the range of $422-470^{\circ} \mathrm{C}$ in air and $457-494^{\circ} \mathrm{C}$ in nitrogen. These polymers were soluble in common polar solvents. In comparison with the soluble aromatic polyimides based on fluorine containing monomers, TCA-AH polyimides showed slightly less thermal stability and lower solubility in specific solvents such as THF.

Acknowledgements. The authors thank Dr. Yoshiyuki Oishi of Iwate University, Dr. Atsushi Takahara of Kyushu University and Dr. Virgil Percec of Case Western Reserve University for valuable advises. Financial support from Technopolis Foundation of Kurume-Tosu is gratefully acknowledged.

\section{REFERENCES}

1. K. L. Mittal, "Polyimides," Plenum Press, New York, N.Y., 1984.

2. J. E. McGrath, M. E. Rogers, C. A. Arnold, Y. J. Kim, and J. C. Hedrick, Macromol. Chem., Macromol. Symp., 51, 103 (1991).

3. S. Z. D. Cheng, F. E. Arnold, Jr., A. Zhang, S. L.-C. Hsu, and F. W. Harris, Macromolecules, 24, 5856 (1991).

4. F. E. Arnold, Jr., S. Z. D. Cheng, S. L.-C. Hsu, C. J. Lee, F. W. Harris, and S.-F. Lau, Polymer, 33, 5179 (1992).

5. M. E. Rogers, M. H. Brink, J. E. McGrath, and A. Brennan, Polymer, 34, 849 (1993).

6. J. W. Park, M. Lee, M.-H. Lee, J. W. Liu, S. D. Kim, J. Y. Chang, and S. B. Rhee, Macromolecules, 27, 3459 (1994).

7. P. A. Falcigno, S. Jasne, and M. King, J. Polym. Sci., Polym. 
Chem. Ed., 30, 1433 (1992).

8. C.-P. Yang and J.-H. Lin, J. Polym. Sci., Polym. Chem. Ed., 32, 423 (1994).

9. Y. Oishi, M. Ishida, M. Kakimoto, Y. Imai, and T. Kurosaki, J. Polym. Sci., Polym. Chem. Ed., 30, 1027 (1992).

10. C. A. Arnold, J. D. Summers, Y. P. Chen, R. H. Bott, D. Chen, and J. E. McGrath, Polymer, 30, 986 (1989).

11. S. Itamura, M. Yamada, S. Tamura, T. Matsumoto, and T. Kurosaki, Macromolecules, 26, 3940 (1993).

12. M. Yamada, M. Kusama, T. Matsumoto, and T. Kurosaki, Macromolecules, 26, 4961 (1993).
13. M. Kusama, T. Matsumoto, and T. Kurosaki, Macromolecules, 27, 1117 (1994).

14. M. Nishikawa, Y. Yokoyama, N. Bessho, D.-S. Seo, Y. Iimura, and S. Kobayashi, Jpn. J. Appl. Phys., 33, L810 (1994).

15. M. Nishikawa, T. Suganuma, Y. Tsuda, N. Bessho, Y. Iimura, and S. Kobayashi, Jpn. J. Appl. Phys., 33, L1113 (1994).

16. M. Nishikawa, T. Miyamoto, S. Kawamura, Y. Tsuda, N. Bessho, D.-S. Seo, Y. Iimura, and S. Kobayashi, Mol. Cryst. Liq. Cryst., 258, 285 (1995).

17. Y. Oishi, M. Ishida, H. Takado, M. Yoneyama, M. Kakimoto, and Y. Imai, J. Polym. Sci., Polym. Chem. Ed., 28, 1763 (1990). 